\title{
LOCAL AND GLOBAL CALIBRATION OF COORDINATE MEASURING MACHINES
}

\author{
S.D. ANTUNES AND P.M.V.C.D. ANTUNES \\ Instituto Superior Técnico, Av ${ }^{a}$ Rovisco Pais, 1049-001 Lisboa, PORTUGAL \\ E-mail: santunes@ist.utl.pt,pantunes@mail.ipq.pt
}

M.A.F. VICENTE

Universidade de Coimbra, Departamento de Matemática da FCTUC, Apartado 3008, 3001-454 Coimbra, PORTUGAL

E-mail: vicente@mat.uc.pt

\begin{abstract}
In the last years, we assisted to the development of new high precision machine tools: the hexapod machines. We tried to use the hexapod machine as an artefact for CMM's calibration. However, given their cost and their resolution, they are not the best solution for a transportable artefact. By modifying the hexapod structure, we develop two different artefacts: one for local CMM's calibration and another one for global calibration.

Local calibration allows us the determination of the transfer function characterizing the sensor displacement of the CMM. This local calibration is based on the measurement of a rigid artefact of a known geometry, derived from hexapod geometry, which allows us to determine the errors of displacement of the sensor of the CMM.

The artefact for global calibration uses a self-calibrated method, based on measurements from three miniature laser interferometers, measuring the position of a sphere in the volume of the CMM.

The paper describes the patented artefacts for local and global calibration, as well as the referring mathematical problems resulting from self-calibration of global artefact and the method of interpretation of the measurement results of the artefact used in the measure of local errors.
\end{abstract}

\section{Introduction}

The modern definition of traceability intimately links the concepts of calibration (i.e., connection to the SI unit) and measurement uncertainty. In a typical coordinate measuring machine (CMM) measurement problem the measurement under consideration bears little resemblance to the measurand or validity conditions of the CMM calibration. Consequently, the metrologist must develop methods to combine the known CMM calibration information together with the measurement specific factors to generate a task specific uncertainty statement ${ }^{1}$.

In the last years, we assisted to the development of new high precision machine tools: the hexapod machines (see figure 1). We tried to use the hexapod machine as

\footnotetext{
${ }^{1}$ Phillips, S.D., Traceability, Calibration, and Measurement Uncertainty Issues Regarding Coordinate Measuring Machines and Other Complex Instruments, Proc. Assoc. for Coordinate Metrology Canada (ACMC), Ontario, Canada, June 2000.
}

Advanced Mathematical and Computational Tools in Metrology V

Edited by P Ciarlini, M G Cox, E Filipe, F Pavese \& D Richter

๑) 2001 World Scientific Publishing Company (pp. 16-22) 
an artefact for CMM's calibration, trying to get a similar method to the method used by T.Takatsuji et al [2]. However, their cost and their resolution make them not the best solution for a transportable artefact. Modifying the hexapod structure, we develop two different artefacts: one for local CMM's calibration and another one for global calibration.

We intend as local errors: errors of rotational type $\left(R_{x} R_{y} R_{z}\right)$ and translational displacement $\left(T_{x} T_{y} T_{z}\right)$, when measuring forms in near points. So, local calibration allows us the determination of the transfer function characterizing the sensor displacement of the CMM. It is based on the measurement of a rigid artefact of a known geometry, derived from hexapod geometry, which allows us to determine the errors of displacement of the sensor of the CMM.

We define as global errors: errors in the determination of sensor coordinates when measuring the center of a sphere with CMM sensor. The artefact for global calibration uses a self-calibrated method, based on measurements of three miniature laser interferometers, measuring the position of a sphere in the volume of the CMM.

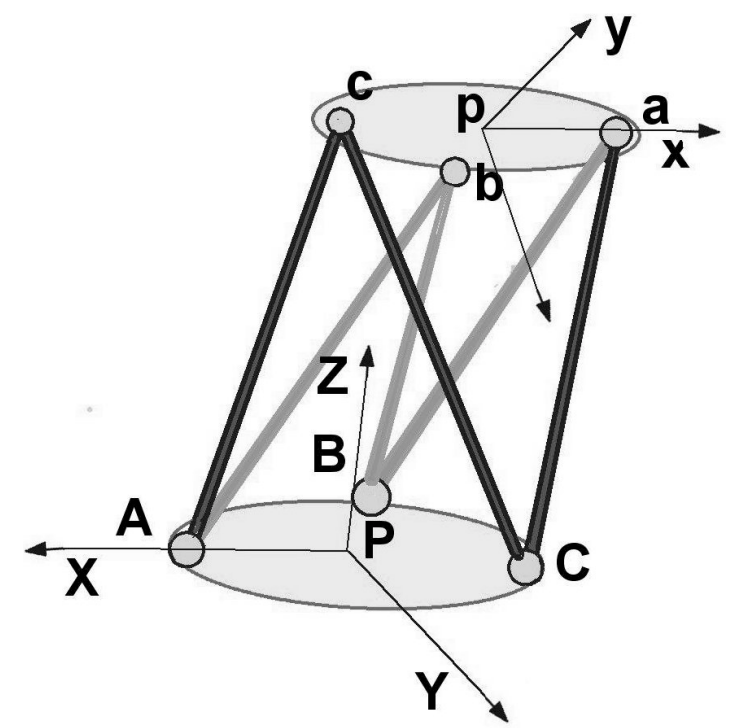

Figure 1. Hexapod machine: measuring the length of the six links $\overline{A c}, \overline{c C}, \overline{C a}, \overline{a B}, \overline{B b}, \overline{b A}$ enables determining the relative position of solids $\overline{P A B C}$ and $\overline{p a b c}$.

\section{Local calibration of CMMs}

A six-sphere artefact composed by two rigid sets of three spheres mounted as a solid structure (see figure 2) has been tested, in order to measure local errors in CMMs. The connection of the two sets of three spheres (of equal or different diameters)

Advanced Mathematical and Computational Tools in Metrology V

Edited by P Ciarlini, M G Cox, E Filipe, F Pavese \& D Richter

(C) 2001 World Scientific Publishing Company (pp. 16-22) 
produces a solid connection with zero degrees of freedom, enabling the mounting and dismounting of the artefact in order to easily measure lower set and upper set of spheres (always mounted in the same relative position).

We can consider the six-sphere artefact as a hexapod system (see figure 3) where the six bars connecting the center of the six spheres consists only in the material of the spheres (The sum of the radius from the lower set spheres with the radius of spheres from upper set gives the length of the bars).

The measuring method is based on the geometry of the artefact, compared with the measured geometry. Measurements are relative to a coordinate referential system centered at the point $P$ (see figure 3 ), with the $\mathrm{Z}$ axis perpendicular to the plane defined by the three center points (points $A, B$ and $C$ ) of the lower set of spheres, and the $\mathrm{X}$ axis passing through the center (point $A$ ) of a reference sphere in the lower set.

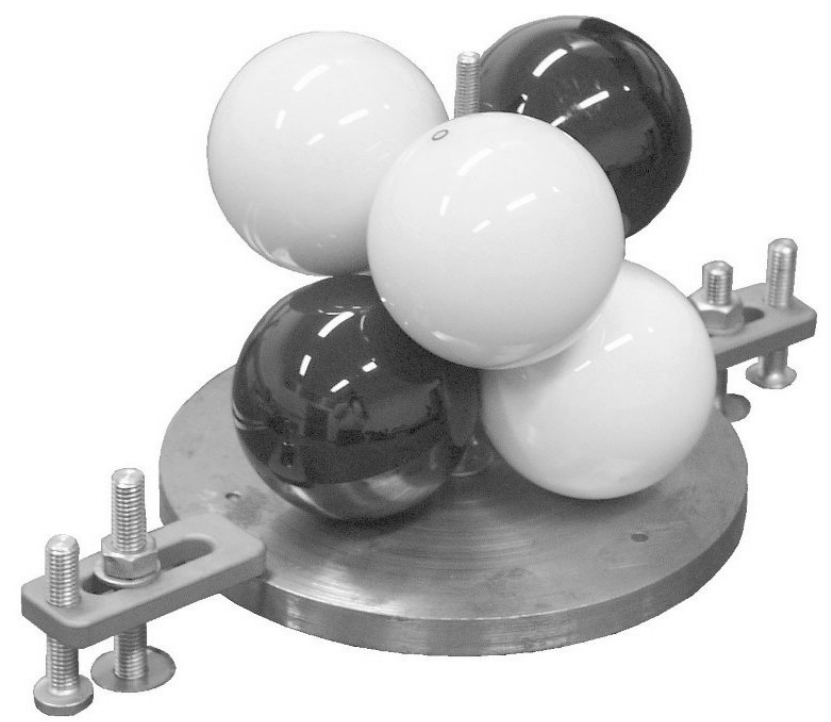

Figure 2. Artefact for measurement of local errors on CMMs.

We don't need to know the geometry of the six-spheres artefact, we can always measure relative errors, referenced to an initial position. The spheres used are industrial spheres and their characteristics (diameter, sphericity, ...) are not too much important. Relative spatial distribution of the measured sphere centers are not influenced by spheres characteristics, it only depends on CMM hard (mechanical) and software errors.

With the previous defined referential and the measured positions of the three centers of the spheres forming the upper set, and comparing results in different positions on the CMM (to the initial position) we can analyze local relative errors of the CMM,

Advanced Mathematical and Computational Tools in Metrology V

Edited by P Ciarlini, M G Cox, E Filipe, F Pavese \& D Richter

(C) 2001 World Scientific Publishing Company (pp. 16-22) 
as well as the relative position of all six spheres (doing and comparing measurements obtained rotating the artefact around his axis $\mathrm{Z}$, on a fixed position).

Rotational and translational components of local errors can be measured comparing the position of the upper set of spheres relatively to the lower set on different positions over the CMM.

On the table 1, we can observe local errors measured on a CMM using this method. The determination of rotational and translational error displacement of CMM sensor plus the software errors, using the six-sphere artefact, is based on the assumption that link variation (geometry of sphere centers measured) is identical to the displacement of the hexapod with identical link variation (the model is obtained linearising hexapod model around the reference position):

$$
\left[R_{x} R_{y} R_{z} T_{x} T_{y} T_{z}\right]^{\mathrm{T}}=\boldsymbol{M} *\left[\delta L_{1} \delta L_{2} \delta L_{3} \delta L_{4} \delta L_{5} \delta L_{6}\right]^{\mathrm{T}},
$$

where $R_{x} R_{y} R_{z} T_{x} T_{y} T_{z}$ are rotational and translational errors; $\delta L_{1} \delta L_{2} \delta L_{3} \delta L_{4} \delta L_{5}$ $\delta L_{6}$ are measured link variations resulting from CMM local errors and $\boldsymbol{M}$ is the operator modeling small variations.

\section{Global calibration of CMMs}

On the hexapod machine represented on figure 1 let us consider the solid $\overline{p a b c}$ as a sphere, in such a way that points $p, a, b$, and $c$ are coincident as indicated in figure 4. Measuring the length of the six links $\overline{A c}, \overline{c C}, \overline{C a}, \overline{a B}, \overline{B b}, \overline{b A}$ on hexapod machine reduces to the measurement of only three links: $\overline{A p}, \overline{B p}, \overline{C p}$.

A self-calibrating system has been developed in such a way that, instead of measuring the three links, we measure the increments for the links with three miniature laser interferometers: position of points $A, B$ and $C$ (center of three similar spheres) are unknown. Moving the sphere centered on $p$ (similar to spheres $A, B$ and $C$ ) on the plane defined by $\overline{A B C}$ and measuring the length variation for the three links enables us to self-calibrate the system identifying coordinates for $A, B$ and $C$, as well as the initial length for the three links $\overline{A p}, \overline{B p}, \overline{C p}$.

Knowing the length of all links, as well as the position of points $A, B$ and $C$ we can also know the position of $p$, center of the moving sphere. Self-calibrating the system on the measurement table of CMM, enables us to know the coordinates of the moving sphere centered on $p$, in the measurement volume of CMM. So, by comparing the calculated position of $p$ (from measurement of the three links by laser interferometry) with the measured position by CMM, the global error of CMM for each position of $p$ can be calculated.

Actually, we have developed a numerical simulation [1], based on data fusion techniques, in order to validate the capability of the artefact to be used as an artefact for global error measurement in CMMs.

Advanced Mathematical and Computational Tools in Metrology V

Edited by P Ciarlini, M G Cox, E Filipe, F Pavese \& D Richter

(C) 2001 World Scientific Publishing Company (pp. 16-22) 


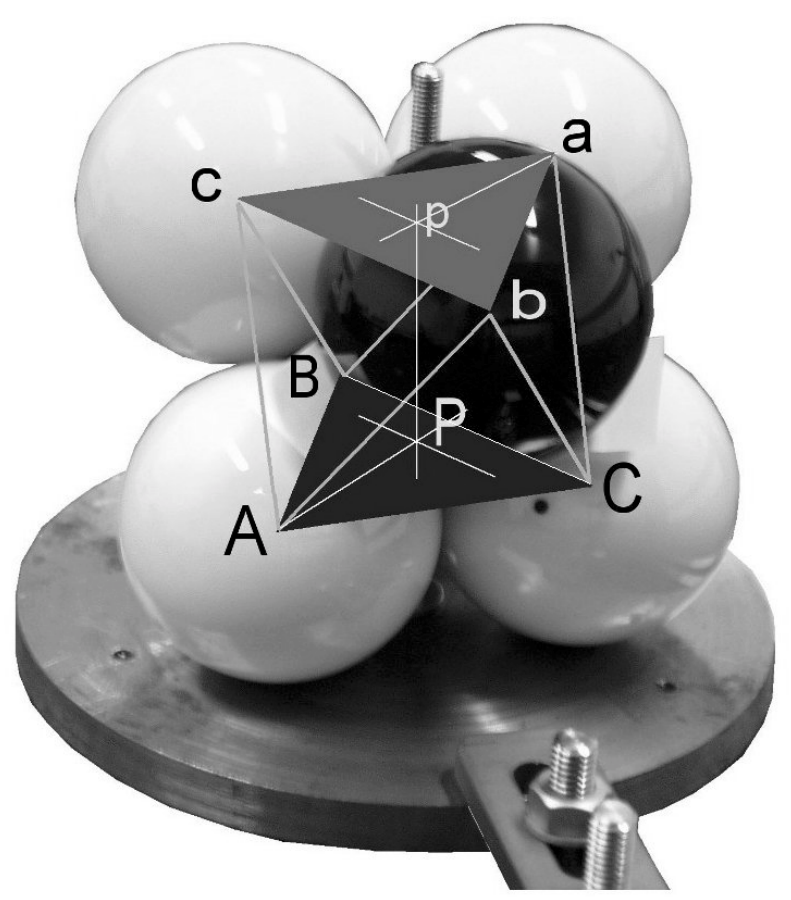

Figure 3. Artefact as an hexapod (measurement of hexapod geometry enables absolute local errors determination if hexapod geometry is known, or relative errors if comparing two measures in different positions).

The artefact is modeled by a set of $m$ (three) equations:

$$
Z_{i j}=\left(x_{0 i}-x_{j}\right)^{2}+\left(y_{0 i}-y_{j}\right)^{2}+\left(z_{0 i}-z_{j}\right)^{2}-D_{i j}^{2}=0,
$$

with:

$\left(x_{0 i}, y_{0 i}, z_{0 i}\right)=$ Coordinates of the fixed sphere, $i=1, m$,

$\left(x_{j}, y_{j}, z_{j}\right)=$ Coordinates of the moving sphere at time $j$,

$D_{i j}=$ Distance, at time $j$, from the moving sphere to the fixed sphere $i$.

Each equation of that type corresponds to one link connecting a fixed sphere to the moving sphere, and all $x_{j}, y_{j}, z_{j}, x_{0 i}, y_{0 i}, z_{0 i}$ are unknowns at time $j . D_{i j}$ are the distances estimated by:

$$
D_{i j}=l_{i}+d l_{i j}
$$

where $d l_{i j}$ represents the distance variations, measured by laser interferometry, of the link that connects the sphere $i$ to the moving sphere at time $j$, and all the values of $l_{i}$ are unknowns corresponding to initial links length.

All fixed spheres are constrained to the CMM table. During self-calibration, the moving sphere is also constrained to the CMM table. Flatness table errors are

Advanced Mathematical and Computational Tools in Metrology V

Edited by P Ciarlini, M G Cox, E Filipe, F Pavese \& D Richter

(C) 2001 World Scientific Publishing Company (pp. 16-22) 
neglected because their contributions for initial link length, so as for link variations, are second order contributions. The position and uncertainty of the fixed spheres are unknowns, so as position and uncertainty for the mobile sphere. Uncertainty will depend on geometry, time of self-calibration and position of mobile sphere. Their values are estimated by data fusion techniques.

Measured values on the artefact:

\begin{tabular}{|c|c|c|c|c|c|c|c|c|}
\hline \multirow{2}{*}{ sphere } & \multicolumn{4}{|c|}{ position A (reference) } & \multicolumn{4}{c|}{ position B } \\
\cline { 2 - 9 } & $\mathbf{X}_{\text {CMM }}$ & $\mathbf{Y}_{\text {CMM }}$ & $\mathbf{Z}_{\text {CMM }}$ & diam & $\mathbf{X}_{\text {CMM }}$ & $\mathbf{Y}_{\text {CMM }}$ & $\mathbf{Z}_{\text {CMM }}$ & diam \\
\hline $\mathbf{1}$ & 756,0800 & 404,6639 & 125,2983 & 61,6014 & 361,2134 & 204,0272 & 125,2926 & 61,6033 \\
\hline $\mathbf{2}$ & 702,9455 & 373,8399 & 125,1831 & 61,4026 & 307,7679 & 173,7528 & 125,1788 & 61,4021 \\
\hline $\mathbf{3}$ & 702,7488 & 435,1779 & 125,1331 & 61,4231 & 308,2029 & 235,0875 & 125,1228 & 61,4226 \\
\hline $\mathbf{4}$ & 684,8597 & 404,4795 & 175,3288 & 61,5907 & 290,0138 & 204,5765 & 175,3206 & 61,5902 \\
\hline $\mathbf{5}$ & 738,0274 & 435,3990 & 175,5098 & 61,5753 & 343,4880 & 234,9618 & 175,5040 & 61,5739 \\
\hline $\mathbf{6}$ & 738,1808 & 373,9441 & 175,5152 & 61,4538 & 343,0252 & 173,5092 & 175,5156 & 61,4522 \\
\hline
\end{tabular}

Measured values referred to lower set referential:

\begin{tabular}{|c|c|c|c|c|c|c|}
\hline sphere & $\bar{x}$ & $\bar{y}$ & $\mathbf{z}$ & $x$ & $\bar{y}$ & $\bar{z}$ \\
\hline 1 & 35,4513 & 0,0000 & 0,0000 & 35,4495 & 0,0000 & 0,0000 \\
\hline 2 & $-17,7683$ & $-30,6771$ & 0,0000 & $-17,7667$ & $-30,6759$ & 0,0000 \\
\hline 3 & $-17,7956$ & 30,6613 & 0,0000 & $-17,7936$ & 30,6603 & 0,0000 \\
\hline 4 & $-35,6372$ & $-0,0285$ & 50,2179 & $-35,6188$ & $-0,0325$ & 50,2175 \\
\hline 5 & 17,6160 & 30,7439 & 50,2838 & 17,6255 & 30,7545 & 50,2871 \\
\hline 6 & 17,5996 & $-30,7112$ & 50,2391 & 17,6254 & $-30,6998$ & 50,2427 \\
\hline $\begin{array}{l}\text { upper set } \\
\text { p center }\end{array}$ & $-0,1484$ & 0,0211 & 50,2469 & $-0,1304$ & 0,0274 & 50,2491 \\
\hline
\end{tabular}

Referential versors of upper set of spheres referenced to lower set referential:

\begin{tabular}{|c|c|c|c|c|c|c|}
\hline \multirow{2}{*}{$\begin{array}{c}\text { local in } \\
\text { analysis }\end{array}$} & \multicolumn{6}{|c|}{ local coordinates on CMM } \\
\cline { 2 - 7 } & 756,0802 & 404,6640 & 125,2989 & 361,2135 & 204,0272 & 125,2927 \\
\hline $\begin{array}{c}\text { measured } \\
\text { positions }\end{array}$ & \multicolumn{3}{|c|}{$\mathbf{A}$ (reference) } & \multicolumn{3}{|c|}{$\mathbf{B}$} \\
\hline $\boldsymbol{R}_{\boldsymbol{x}}$ axis & $-1,0000$ & $-0,0014$ & $-0,0008$ & $-1,0000$ & $-0,0017$ & $-0,0009$ \\
\hline $\boldsymbol{R}$ y axis & $-0,0014$ & 1,0000 & $-0,0007$ & $-0,0017$ & 1,0000 & $-0,0007$ \\
\hline $\boldsymbol{R} \mathbf{z}$ axis & 0,0008 & 0,0007 & 1,0000 & 0,0009 & 0,0007 & 1,0000 \\
\hline $\boldsymbol{T}$ (point p) & $-0,1484$ & 0,0211 & 50,2469 & $-0,1304$ & 0,0274 & 50,2491 \\
\hline
\end{tabular}

Local errors:

\begin{tabular}{|c|c|c|c|c|}
\hline \multicolumn{2}{|c|}{ error components } & $\mathrm{X}$ & $\mathrm{Y}$ & $\mathrm{Z}$ \\
\hline \multirow{2}{*}{$\begin{array}{c}\text { rotational } \\
\text { (AB) (rad) }\end{array}$} & $\boldsymbol{R}_{\mathbf{X}}$ axis & 0,0000 & $-0,0003$ & $-0,0001$ \\
\cline { 2 - 5 } & $R_{\text {y } \text { axis }}$ & $-0,0003$ & 0,0000 & 0,0000 \\
\hline $\begin{array}{c}\text { translational }(\mathrm{AB}) \\
\text { (mm) }\end{array}$ & 0,0001 & 0,0000 & 0,0000 \\
\hline
\end{tabular}

Table 1. Example of relative local errors measurement on one CMM.

Advanced Mathematical and Computational Tools in Metrology V

Edited by P Ciarlini, M G Cox, E Filipe, F Pavese \& D Richter

(C) 2001 World Scientific Publishing Company (pp. 16-22) 


\section{Conclusions}

Two different types of artefacts based on hexapod machine have been developed: one to measure local errors on CMMs based on a set of six spheres (two rigid sets of three similar spheres), and another to measure global errors on CMMs based on a set of four independent spheres (being one of them a moving sphere connected by telescopic links to three fixed spheres).

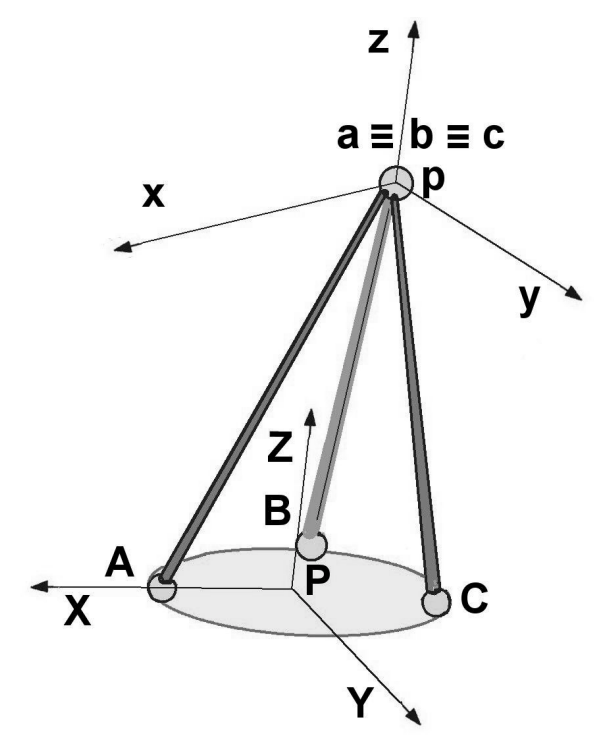

Figure 4. Artefact for global errors measurement.

Measurements with the artefacts have errors and uncertainty unknown. Repetitive measurements and self-calibration enables us to get, during calibration, the errors and an uncertainty changing with self-calibration along time and position. We believe that the developed artefacts enable measurement of local and global CMMs errors. Those artefacts can be applied in many other fields for instance to perform tests of robots, or to act as components of machines or even as CMMs.

\section{References}

1. Antunes, S.D. and Vicente, M.A.F. - Artefact validation by data fusion techniques, Data Fusion SIG, at AMCTM 2000 (Caparica 2000).

2. Takatsuji, T. et al, The first measurement of a three-dimensional coordinate by use of a laser tracking interferometer system based on trilateration, Measurement Science and Technology 9, 1 (1998) pp 38-41.

Advanced Mathematical and Computational Tools in Metrology V

Edited by P Ciarlini, M G Cox, E Filipe, F Pavese \& D Richter

(C) 2001 World Scientific Publishing Company (pp. 16-22) 